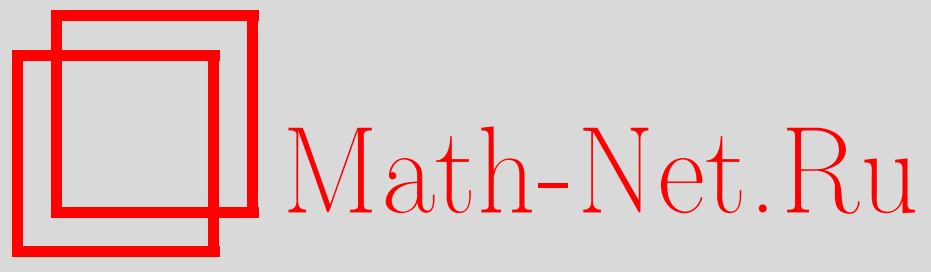

Г. И. Ботиров, У. А. Розиков, Модель Поттса с конкурирующими взаимодействиями на дереве Кэли: контурный метод, ТМФ, 2007, том 153, номер 1, 86-97

DOI: https://doi.org/10.4213/tmf6123

Использование Общероссийского математического портала Math-Net.Ru подразумевает, что вы прочитали и согласны с пользовательским соглашением http://www . mathnet.ru/rus/agreement

Параметры загрузки:

IP : 54.196 .121 .252

26 апреля 2023 г., 17:00:13

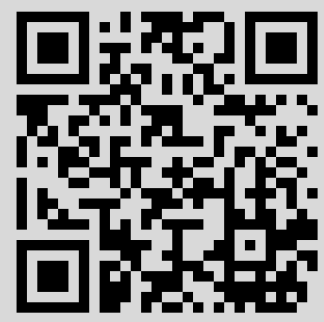




\title{
МОДЕЛЬ ПОТТСА С КОНКУРИРУЮЩИМИ ВЗАИМОДЕЙСТВИЯМИ НА ДЕРЕВЕ КЭЛИ: КОНТУРНЫЙ МЕТОД
}

\begin{abstract}
Рассматривается модель Поттса с тремя значениями спина и с конкурирующими взаимодействиями радиуса $r=2$ на дереве Кэли порядка $k=2$. Дается полное описание основных состояний этой модели. При помощи контурного метода на дереве доказано, что при достаточно низких температурах эта модель имеет три различные гиббсовские меры.
\end{abstract}

Ключевые слова: дерево Кэли, конфигурация, контурный метод, основные состояния, мера Гиббса.

\section{1. ВВЕДЕНИЕ}

Эта работа является продолжением работ [1], [2], посвященных развитию контурного метода на дереве Кэли. Контурный метод на решетке $\mathbb{Z}^{d}, d \geqslant 1$, имеет общее название теории Пирогова-Синая [3]-[8]. Теория Пирогова-Синая обобщает технику Пайерлса [9], примененную для модели Изинга на $\mathbb{Z}^{2}$.

В работах [10]-[13] различные модели на дереве Кэли изучены с применением метода марковских случайных полей и рекуррентных уравнений. Этот метод не всегда удобен, и поэтому естественно развитие теории Пирогова-Синая на дереве Кэли.

В работе [1] эта теория развита для $q$-компонентной модели (с взаимодействиями ближайшего соседа), а в работе [2] - для модели Изинга с конкурирующими взаимодействиями с радиусом взаимодействия $r=2$. Настоящая работа обобщает результаты работы [2] для модели Поттса с конкурирующими взаимодействиями.

Структура работы следующая. В разделе 2 даются основные определения. В разделе 3 описываются все периодические и некоторые непериодические основные состояния рассматриваемой модели. Определение и некоторые свойства контура даны в разделе 4 . В разделе 5 с помощью контурного метода доказывается неединственность меры Гиббса.

*Национальный университет Узбекистана им. М. Улугбека, Ташкент, Узбекистан. E-mail: botirovg@yandex.ru

${ }^{\dagger}$ Институт математики им. В. И. Романовского, Ташкент, Узбекистан. E-mail: rozikovu@yandex.ru, rozikovu@mail.ru 


\section{2. ОПРЕДЕЛЕНИЯ}

2.1. Дерево Кэли. Дерево Кэли $\Im^{k}$ (см. [14]) порядка $k \geqslant 1$ представляет собой бесконечное дерево, т.е. граф без циклов, из каждой вершины которого выходит ровно $k+1$ ребер. Пусть $\Im^{k}=(V, L, i)$, где $V$ - множество вершин $\Im^{k}, L-$ его множество ребер, $i$ - функция инцидентности, сопоставляющая каждому ребру $l \in L$ его концевые точки $x, y \in V$. Если $i(l)=\{x, y\}$, то вершины $x$ и $y$ называются ближайшими соседями и мы пишем $l=\langle x, y\rangle$. Расстояние $d(x, y), x, y \in V$, на дереве Кэли определяется формулой

$$
\begin{array}{r}
d(x, y)=\min \left\{d \mid \exists x=x_{0}, x_{1}, \ldots, x_{d-1}, x_{d}=y \in V\right. \\
\text { такое, что } \left.\left\langle x_{0}, x_{1}\right\rangle, \ldots,\left\langle x_{d-1}, x_{d}\right\rangle\right\} .
\end{array}
$$

Для фиксированного $x^{0} \in V$ обозначим

$$
\begin{gathered}
W_{n}=\left\{x \in V \mid d\left(x, x^{0}\right)=n\right\}, \quad V_{n}=\left\{x \in V \mid d\left(x, x^{0}\right) \leqslant n\right\}, \\
L_{n}=\left\{l=\langle x, y\rangle \in L \mid x, y \in V_{n}\right\} .
\end{gathered}
$$

Известно, что существует взаимно однозначное соответствие между множеством $V$ вершин дерева Кэли порядка $k \geqslant 1$ и группой $G_{k}$ свободных произведений $k+1$ циклической группы второго порядка с образующими $a_{1}, a_{2}, \ldots, a_{k+1}$.

2.2. Пространство конфигурации и модель. Мы рассматриваем модели, где спин принимает значения из множества $\Phi=\{1,2, \ldots, q\}, q \geqslant 2$. Конфигурация $\sigma$ на множестве $V$ тогда определяется как функция $x \in V \rightarrow \sigma(x) \in \Phi$; множество всех конфигураций совпадает с $\Omega=\Phi^{V}$.

Предположим, что на $\Omega$ действует группа пространственных переходов. Определим периодическую конфигурацию $F_{k}$ как конфигурацию $\sigma(x)$, которая является инвариантной относительно подгруппы $F_{k} \subset G_{k}$ конечного индекса, т.е. $\sigma(y x)=\sigma(x)$ для любых $x \in G_{k}, y \in F_{k}$. Для данной периодической конфигурации индекс подгруппы называется периодом конфигурации. А конфигурация, которая является инвариантной относительно всех переходов, называется трансляиионно-инвариантной.

Гамильтониан модели Поттса с конкурирующими взаимодействиями имеет вид

$$
H(\sigma)=J_{1} \sum_{\substack{\langle x, y\rangle \\ x, y \in V}} \delta_{\sigma(x) \sigma(y)}+J_{2} \sum_{\substack{x, y \in V: \\ d(x, y)=2}} \delta_{\sigma(x) \sigma(y)},
$$

где $J_{1}, J_{2} \in \mathbb{R}$,

$$
\delta_{u v}= \begin{cases}1, & u=v \\ 0, & u \neq v\end{cases}
$$

\section{3. ОСНОВНЫЕ СОСТОЯНИЯ}

Основные состояния для моделей, определенных на $\mathbb{Z}^{d}$, можно найти, например, в [3], [4], [6], [15], [16]. 
Для пары конфигураций $\sigma$ и $\varphi$, совпадающих почти всюду, т.е. всюду, за исключением конечного числа точек, мы рассматриваем относительный гамильтониан $H(\sigma, \varphi)$ различия между энергиями конфигураций $\sigma$ и $\varphi$, т.е.

$$
H(\sigma, \varphi)=J_{1} \sum_{\substack{\langle x, y\rangle \\ x, y \in V}}\left(\delta_{\sigma(x) \sigma(y)}-\delta_{\varphi(x) \varphi(y)}\right)+J_{2} \sum_{\substack{x, y \in V: \\ d(x, y)=2}}\left(\delta_{\sigma(x) \sigma(y)}-\delta_{\varphi(x) \varphi(y)}\right),
$$

где $J=\left(J_{1}, J_{2}\right) \in \mathbb{R}^{2}$ - произвольный фиксированный параметр.

Пусть $M$ - множество единичных шаров с вершинами в $V$. Будем называть сужение конфигурации $\sigma$ на шаре $b \in M$ ограниченной конфигурацией $\sigma_{b}$. Определим энергию конфигурации $\sigma_{b}$ на шаре $b$ следующим образом:

$$
U\left(\sigma_{b}\right) \equiv U\left(\sigma_{b}, J\right)=\frac{1}{2} J_{1} \sum_{\substack{\langle x, y\rangle \\ x, y \in b}} \delta_{\sigma(x) \sigma(y)}+J_{2} \sum_{\substack{x, y \in b: \\ d(x, y)=2}} \delta_{\sigma(x) \sigma(y)},
$$

где $J=\left(J_{1}, J_{2}\right) \in \mathbb{R}^{2}$.

Легко доказать следующую лемму.

Лемма 1. Относительный гамильтониан (3) имеет вид

$$
H(\sigma, \varphi)=\sum_{b \in M}\left(U\left(\sigma_{b}\right)-U\left(\varphi_{b}\right)\right)
$$

Рассмотрим случай $k=2$ и $q=3$. Легко видеть, что $U\left(\sigma_{b}\right) \in\left\{U_{1}, \ldots, U_{6}\right\}$ для любого $\sigma_{b}$, где

$$
\begin{aligned}
& U_{1}=\frac{3}{2} J_{1}+3 J_{2}, \quad U_{2}=J_{1}+J_{2}, \quad U_{3}=3 J_{2}, \\
& U_{4}=\frac{1}{2} J_{1}, \quad U_{5}=J_{2}, \quad U_{6}=\frac{1}{2} J_{1}+J_{2} .
\end{aligned}
$$

ОПРЕДЕЛЕНИЕ 1. Конфигурация $\varphi$ называется основным состоянием относительного гамильтониана $H$, если $U\left(\varphi_{b}\right)=\min \left\{U_{1}, U_{2}, \ldots, U_{6}\right\}$ для любого $b \in M$.

Обозначим $C_{i}=\left\{\sigma_{b}: U\left(\sigma_{b}\right)=U_{i}\right\}$ и $U_{i}(J)=U\left(\sigma_{b}, J\right)$, если $\sigma_{b} \in C_{i}, i=1,2, \ldots, 6$.

Tеорема 1. А. Для любого класса $C_{i}, i=1,2,3,4$, и для любой ограниченной конфигурачии $\sigma_{b} \in C_{i}$ существует периодическая конфигурация $\varphi$ (на дереве Кэли) c периодом $p \leqslant 6$ такая, что $\varphi_{b^{\prime}} \in C_{i}$ для любого $b^{\prime} \in M u \varphi_{b}=\sigma_{b}$.

Б. Для любого $\sigma_{b} \in C_{5}$ существует конфигураиия $\varphi$ (вообще говоря, не являющаяся периодической) на дереве Кэли такая, что $\varphi_{b^{\prime}} \in C_{5}$ для любого $b^{\prime} \in M u$ $\varphi_{b}=\sigma_{b}$.

Доказательство. А. Для произвольного класса $C_{i}, i=1,2,3,4$, и $\sigma_{b} \in C_{i}$ построим конфигурацию $\varphi$ следующим образом: без ограничения общности мы можем в качестве шара $b$ взять шар с центром $e \in G_{2}$ (где $e-$ единичный элемент $\left.G_{2}\right)$, т.е. $b=\left\{e, a_{1}, a_{2}, a_{3}\right\}$.

Рассмотрим несколько случаев. 
Случай $C_{1}$. В этом случае мы имеем $\sigma(x)=i, i=1,2,3$, для любого $x \in b$. Таким образом, конфигурация $\varphi$ совпадает с трансляционно-инвариантной, т.е. $\varphi^{(i)}=$ $\left\{\varphi^{(i)}(x)=i\right\}$, где $i=1,2,3$.

Случай $C_{2}$. Рассмотрим $H_{2}=\left\{x \in G_{2}: \omega_{2}(x)\right.$ четно $\}$, где $\omega_{2}(x)$ - число букв $a_{2}$ в слове $x \in G_{2}$. Отметим (см. [17]), что $H_{2}$ - нормальная подгруппа индекса 2 для $G_{2}$. По построению мы имеем $\sigma_{b}(x)=i$ для любого $x \in b \cap H_{2}$ и $\sigma(x)=j$ для любого $x \in b \cap\left(G_{2} \backslash H_{2}\right)$, где $i, j=1,2,3, i \neq j$.

Продолжим ограниченную конфигурацию $\sigma_{b} \in C_{i}$ на целую решетку $\Im^{2}$ (которую мы обозначаем $\left.\varphi^{(i j)}\right)$ следующим образом:

$$
\varphi^{(i j)}(x)= \begin{cases}i, & x \in H_{2}, \\ j, & x \in G_{2} \backslash H_{2}\end{cases}
$$

где $i, j=1,2,3, i \neq j$.

Случай $C_{3}$. В этом случае рассмотрим $H_{3}=\left\{x \in G_{2}:|x|\right.$ четно $\}$, где $|x|$ - длина слова $x$. Отметим, что $H_{3}$ является также нормальной подгруппой группы $G_{2}$ индекса 2. В этом случае конфигурация $\psi^{(i j)}$ может быть определена как

$$
\psi^{(i j)}(x)= \begin{cases}i, & x \in H_{3}, \\ j, & x \in G_{2} \backslash H_{3}\end{cases}
$$

где $i, j=1,2,3, i \neq j$.

Таким образом, мы получаем периодическую конфигурацию $\psi^{(i j)}$ с периодом $p=2$ (равным индексу подгруппы); ясно, что $\psi_{b}^{(i j)}=\sigma_{b}$.

Случай $C_{4}$. Пусть $S_{3}$ - группа перестановок третьего порядка. Возьмем $\pi_{0}, \pi_{1}$, $\pi_{2} \in S_{3}$ такие, что

$$
\pi_{0}=\left(\begin{array}{lll}
1 & 2 & 3 \\
1 & 2 & 3
\end{array}\right), \quad \pi_{1}=\left(\begin{array}{lll}
1 & 2 & 3 \\
2 & 1 & 3
\end{array}\right), \quad \pi_{2}=\left(\begin{array}{lll}
1 & 2 & 3 \\
1 & 3 & 2
\end{array}\right) .
$$

Легко видеть, что $\pi_{0}=\pi_{1}^{2}=\pi_{2}^{2}$. Рассмотрим отображение $u:\left\{a_{1}, a_{2}, a_{3}\right\} \mapsto\left\{\pi_{0}\right.$, $\left.\pi_{1}, \pi_{2}\right\}$ :

$$
u\left(a_{i}\right)= \begin{cases}\pi_{0}, & i=3, \\ \pi_{i}, & i=1,2,\end{cases}
$$

и пусть функция $f: G_{2} \mapsto S_{3}$ определена следующим образом:

$$
f(x)=f\left(a_{i_{1}} a_{i_{2}} \ldots a_{i_{n}}\right)=u\left(a_{i_{1}}\right) \ldots u\left(a_{i_{n}}\right) .
$$

Заметим (см. [17]), что $H_{4}=\left\{x \in G_{2}: f(x)=\pi_{0}\right\}$ - нормальная подгруппа с индексом 6 . Пусть $G_{2} / H_{4}=\left\{\aleph_{0}, \ldots, \aleph_{5}\right\}$ - факторгруппа. В этом случае мы определяем конфигурацию $\varphi^{(i j p)}$ следующим образом:

$$
\varphi^{(i j p)}(x)= \begin{cases}i, & x \in \aleph_{0} \cup \aleph_{3}, \\ j, & x \in \aleph_{1} \cup \aleph_{5}, \\ p, & x \in \aleph_{2} \cup \aleph_{4} .\end{cases}
$$


Таким образом, мы получаем периодическую конфигурацию $\varphi^{(i j p)}$ с периодом $p=6$, со свойством $\varphi_{b}^{(i j p)}=\sigma_{b}$.

Б. Случай $C_{5}$. Для $x \in G_{2}$ введем обозначения $l(x)=l\left(a_{i_{1}} \ldots a_{i_{n}}\right)=i_{1}, r(x)=$ $r\left(a_{i_{1}} \ldots a_{i_{n}}\right)=i_{n}$. В этом случае $\psi^{(i j p)}$ может быть определена как

$$
\psi^{(i j p)}(x)= \begin{cases}i, & r(x)=1, \quad l(x) \neq 3 \quad \text { или } \quad r(x)=3, \quad l(x)=3, \\ j, & r(x)=2, \\ p, & r(x)=3, \quad l(x) \neq 3 \quad \text { или } \quad r(x)=1, \quad l(x)=3, \\ p, & x=e .\end{cases}
$$

Легко видеть (см. [2]), что для каждой конфигурации $\varphi$ мы имеем $\varphi_{b} \in C_{i}, i=$ $1,2,3,4,5$, для любого $b \in M$.

Для каждого $m=1,2,3,4,5,6$ обозначим через $A_{m}$ множество точек $J$ таких, что

$$
A_{m}(J)=\left\{J \in \mathbb{R}^{2}: \min \left\{U_{1}(J), U_{2}(J), \ldots, U_{6}(J)\right\}=U_{m}\right\} .
$$

Легко видеть, что

$$
\begin{aligned}
& A_{1}=\left\{J \in \mathbb{R}^{2}: J_{1} \leqslant 0, J_{1}+4 J_{2} \leqslant 0\right\}, \\
& A_{2}=\left\{J \in \mathbb{R}^{2}: J_{2} \geqslant 0,-4 J_{2} \leqslant J_{1} \leqslant-2 J_{2}\right\}, \\
& A_{3}=\left\{J \in \mathbb{R}^{2}: J_{1} \geqslant 0, J_{2} \leqslant 0\right\}, \\
& A_{4}=\left\{J \in \mathbb{R}^{2}: J_{2} \geqslant 0,-2 J_{2} \leqslant J_{1} \leqslant 2 J_{2}\right\}, \\
& A_{5}=\left\{J \in \mathbb{R}^{2}: J_{2} \geqslant 0, J_{1}-2 J_{2} \geqslant 0\right\}, \\
& A_{6}=\left\{J \in \mathbb{R}^{2}: J_{1}=0, J_{2}=0\right\},
\end{aligned}
$$

и $\mathbb{R}^{2}=\bigcup_{i=1}^{6} A_{i}$.

ЗАмечАниЕ. Так как в случае $A_{6}$ нет никакого взаимодействия, то мы в дальнейшем не рассматриваем класс $C_{6}$. По этой же причине этот класс также не включен в теорему 1 .

Обозначим $B=A_{1} \cap A_{3}, B_{0}=A_{1} \cap A_{2}, B_{1}=A_{2} \cap A_{4}, B_{2}=A_{4} \cap A_{5}, B_{3}=A_{5} \cap A_{3}$, $\tilde{A}_{1}=A_{1} \backslash\left(B \cup B_{0}\right), \tilde{A}_{2}=A_{2} \backslash\left(B_{0} \cup B_{1}\right), \tilde{A}_{3}=A_{3} \backslash\left(B \cup B_{2}\right), \tilde{A}_{4}=A_{4} \backslash\left(B_{1} \cup B_{2}\right)$, $\tilde{A}_{5}=A_{5} \backslash\left(B_{2} \cup B_{3}\right)$. Пусть $G S(H)$ - множество всех основных состояний, а $G S_{\mathrm{p}}(H)$ множество всех периодических основных состояний.

Teоpema 2. А. Eсли $J=(0,0), \operatorname{mo} G S(H)=\Omega$.

Б. 1. Если $J \in \tilde{A}_{1}, \operatorname{mo} G S_{\mathrm{p}}(H)=\left\{\varphi^{(i)}: i=1,2,3\right\}$.

2. Если $J \in \tilde{A}_{2}$, mo $G S_{\mathrm{p}}(H)=\left\{\varphi^{(i j)}: i, j=1,2,3, i \neq j\right\},\left|G S_{\mathrm{p}}(H)\right|=3$.

3. Eсли $J \in \tilde{A}_{3}, \operatorname{mo} G S_{\mathrm{p}}(H)=\left\{\psi^{(i j)}: i, j=1,2,3, i \neq j\right\},\left|G S_{\mathrm{p}}(H)\right|=3$.

4. Если $J \in \tilde{A}_{4}$, mo $G S_{\mathrm{p}}(H)=\left\{\varphi^{(i j p)}: i, j, p=1,2,3, i \neq j \neq p\right\},\left|G S_{\mathrm{p}}(H)\right|=3$.

5. Если $J \in \tilde{A}_{5}$, mo $G S(H) \supset\left\{\psi^{(i j p)}: i, j, p=1,2,3, i \neq j \neq p\right\}$, где длякаждого случал конфигурачии $\varphi^{(i)}, \varphi^{(i j)}, \psi^{(i j)}, \varphi^{(i j p)}, \psi^{(i j p)}$ определены в доказательстве теоремы 1. 
B. 1 Если $J \in B_{i} \backslash\{(0,0)\}, i=0,1,2,3$, mo $\left|G S_{\mathrm{p}}(H)\right|=6$.

2. Если $J \in B \backslash\{(0,0)\}, m o\left|G S_{\mathrm{p}}(H)\right|=6$.

Кроме того, если $J \in \mathbb{R}^{2} \backslash B$, то есть по крайней мере счетное множество непериодических основных состояний.

ДокАЗАТЕльство. Случай А тривиален. В случаях Б и В для данной конфигурации $\sigma_{b}$, которая делает энергию $U\left(\sigma_{b}\right)$ минимальной, с помощью теоремы 1 можно построить периодические конфигурации $\varphi^{(i j)}, \varphi^{(i j p)}$ (с периодом $p \leqslant 6$ ). Для каждого случая точное число таких основных состояний совпадает с числом различных конфигураций $\varphi_{b}$ таких, что энергия $U\left(\varphi_{b}\right)$ минимальна для любого $b \in M$.

ОПРЕДЕЛЕНИЕ 2. Пусть $G S(H)$ - множество всех возможных основных состояний относительного гамильтониана $H$. Шар $b \in M$ назовем вырожденным шаром конфигурации $\sigma$, если $\sigma_{b} \neq \varphi_{b}$ для всех $\varphi \in G S(H)$. Множество всех вырожденных шаров конфигурации $\sigma$ называется гранищей конфигуращии и обозначается $\partial(\sigma)$.

ОПРЕДЕЛЕНИЕ 3. Относительный гамильтониан $H$ с множеством основных состояний $G S(H)$ удовлетворяет условию Пайерлса, если для любого $\varphi \in G S(H)$ и для всех конфигураций $\sigma$, совпадающих почти всюду с $\varphi$, имеет место

$$
H(\sigma, \varphi) \geqslant \lambda|\partial(\sigma)|
$$

где $\lambda$ является положительной постоянной, которая не зависит от $\sigma$, и $|\partial(\sigma)|$ - число единичных шаров в $\partial(\sigma)$.

Аналогично доказательству теоремы 9 в работе [2] можно доказать следующую теорему.

Теорема 3. Если $J \neq(0,0)$, то условие Пайерлса выполняется.

\section{4. КОНТУРЫ НА ДЕРЕВЕ КЭЛИ}

Рассмотрим случай $J \in \tilde{A}_{1}$, т.е. случай, когда только постоянные конфигурации являются основными состояниями гамильтониана.

Пусть $\Lambda \subset V$ - конечное множество, $\Lambda^{\prime}=V \backslash \Lambda$ и $\omega_{\Lambda}=\left\{\omega(x), x \in \Lambda^{\prime}\right\}, \sigma_{\Lambda}=$ $\{\sigma(x), x \in V\}$ - данные конфигурации. Энергия конфигурации $\sigma_{\Lambda}$ имеет вид

$$
\begin{aligned}
H_{\Lambda}\left(\sigma_{\Lambda} \mid \omega_{\Lambda^{\prime}}\right)=J_{1} & \sum_{\substack{\langle x, y\rangle \\
x, y \in \Lambda}} \delta_{\sigma(x) \sigma(y)}+J_{1} \sum_{\substack{\langle x, y\rangle \\
x \in \Lambda, y \in \Lambda^{\prime}}} \delta_{\sigma(x) \omega(y)}+ \\
& +J_{2} \sum_{\substack{x, y \in \Lambda: \\
d(x, y)=2}} \delta_{\sigma(x) \sigma(y)}+J_{2} \sum_{\substack{x \in \Lambda, y \in \Lambda^{\prime}: \\
d(x, y)=2}} \delta_{\sigma(x) \omega(y)} .
\end{aligned}
$$

Пусть $\omega_{\Lambda^{\prime}}^{(i)} \equiv i, i=1, \ldots, q,-$ постоянные конфигурации вне $\Lambda$. Для каждой конфигурации $\sigma_{\Lambda}$ внутри $\Lambda$ доопределим конфигурации ко всему дереву с $i$-й постоянной конфигурацией и обозначим эту конфигурацию $\sigma_{\Lambda}^{(i)}$, и пусть $\Omega_{\Lambda}^{(i)}=\left\{\sigma_{\Lambda}^{(i)}\right\}$. 
Рассмотрим $V_{n}$ и для данной конфигурации $\sigma_{\Lambda}^{(i)} \in \Omega_{\Lambda}^{(i)}, \Lambda \subset V_{n}$, обозначим $V_{n}^{(j)} \equiv$ $V_{n}^{(j)}\left(\sigma_{\Lambda}^{(i)}\right)=\left\{t \in V_{n}: \sigma_{\Lambda}^{(i)}(t)=j\right\}, j=1, \ldots, q, j \neq i$. Пусть $G^{n, j}=\left(V_{n}^{(j)}, L_{n}^{(j)}\right)$ - граф такой, что

$$
L_{n}^{(j)}=\left\{l=\langle x, y\rangle \in L: x, y \in V_{n}^{(j)}\right\}, \quad j=1, \ldots, q .
$$

Очевидно, что для фиксированного $n$ граф $G^{n, j}$ содержит конечное число (равное $m$ ) максимальных связанных подграфов $G_{r}^{n, j}$, т.е.

$$
G^{n, j}=\left\{G_{1}^{n, j}, \ldots, G_{m}^{n, j}\right\}, \quad G_{r}^{n, j}=\left(V_{n, r}^{(j)}, L_{n, r}^{(j)}\right), \quad r=1, \ldots, n,
$$

где $V_{n, r}^{(j)}-$ множество вершин, $L_{n, r}^{(j)}-$ множество ребер $G_{r}^{n, j}$.

Число элементов множества $A$ обозначим $|A|$.

Два ребра $l_{1}, l_{2} \in L, l_{1} \neq l_{2}$, называются ближсайшими соседними ребрами, если $\left|i\left(l_{1}\right) \cap i\left(l_{2}\right)\right|=1$, и мы пишем в этой случае $\left\langle l_{1}, l_{2}\right\rangle_{1}$.

Для любого связанного компонента $K \subset \Im^{k}$ обозначим через $E(K)$ множество ребер $K$ и

$$
b(K)=\left\{l \in L \backslash E(K): \exists l_{1} \in E(K) \quad \text { такое, что }\left\langle l, l_{1}\right\rangle_{1}\right\} .
$$

ОПРедЕЛЕНиЕ 4. Ребро $l=\langle x, y\rangle \in L_{n+1}$ называется граничным ребром конфигурации $\sigma_{V_{n}}^{(i)}$, если $\sigma_{V_{n}}^{(i)}(x) \neq \sigma_{V_{n}}^{(i)}(y)$. Множество граничных ребер конфигурации называется реберной границей $\Gamma\left(\sigma_{V_{n}}^{(i)}\right) \equiv \Gamma$ этой конфигурации.

Граница Г состоит из $q(q-1) / 2$ частей

$$
\Gamma_{\epsilon}\left(\sigma_{V_{n}}^{(i)}\right) \equiv \Gamma_{\epsilon}, \quad \epsilon \in\{i j: i<j ; i, j=1, \ldots, q\} \equiv Q_{q},
$$

где, например, $\Gamma_{12}$ - множество ребер $l=\langle x, y\rangle$ с $\sigma(x)=1$ и $\sigma(y)=2$.

Подконтуром границы Г называются (конечные) множества $b\left(G_{r}^{n, j}\right), j=1, \ldots, q$, $j \neq i ; r=1, \ldots, m$ (для каждого ребра этого набора вместе с информацией о том, какую часть границы $\Gamma_{\epsilon}, \epsilon \in Q_{q}$, содержит это ребро).

Множества $V_{n, r}^{(j)}, j=1, \ldots, q, j \neq i ; r=1, \ldots, m$, называются интеръером Int $b\left(G_{r}^{n, j}\right)$ подконтура $b\left(G_{r}^{n, j}\right)$.

Пусть $G$ - граф. Обозначим множество вершин и ребер графа $G$ через $V(G)$ и $E(G)$, соответственно. Для любых двух подконтуров $T_{1}, T_{2} \operatorname{paccтояние~} \operatorname{dist}\left(T_{1}, T_{2}\right)$ определяем следующим образом:

$$
\operatorname{dist}\left(T_{1}, T_{2}\right)=\min _{\substack{x \in V\left(T_{1}\right) \\ y \in V\left(T_{2}\right)}} d(x, y),
$$

где $d(x, y)$ - расстояние между точками $x, y \in V$.

ОПРЕДЕЛЕНИЕ 5. Подконтуры $T_{1}, T_{2}$ называются смежными, если $d\left(T_{1}, T_{2}\right) \leqslant 2$. Совокупность подконтуров $A$ называется связанной, если для любых двух подконтуров $T_{1}, T_{2} \in A$ существуют подконтуры $\widetilde{T}_{1}=T_{1}, \widetilde{T}_{2}, \ldots, \widetilde{T}_{l}=T_{2}$ в множестве $A$ такие, что для каждого $i=1, \ldots, l-1$ подконтуры $\widetilde{T}_{i}$ и $\widetilde{T}_{i+1}$ являются смежными. 
ОпРЕДЕЛЕНиЕ 6. Любой максимальный связанный набор (компонент) подконтуров называется контуром границы Г.

Пусть $\gamma=\left\{\gamma_{r}, r=1,2, \ldots\right\}-$ контур границы $\Gamma, \gamma_{r}$ - подконтур. Обозначим

$$
\operatorname{Int} \gamma=\bigcup_{j} \operatorname{Int} \gamma_{j}, \quad \operatorname{imp} \gamma=\{b \in \partial: b \cap \gamma \neq \varnothing\}, \quad|\gamma|=|\operatorname{imp} \gamma| .
$$

Для $A \subset V$ обозначим

$$
\begin{aligned}
B(A) & =\{b \in M: b \cap A \neq \varnothing\}, \\
D_{\operatorname{int}}(A) & =\{x \in A: \exists \quad \text { точка } \quad y \in V \backslash A \text { такая, что }\langle x, y\rangle\}, \\
D(A) & =\{x \in V \backslash A: \exists \quad \text { точка } y \in A \text { такая, что }\langle x, y\rangle\} .
\end{aligned}
$$

Лемма 2. Пусть $K$ - связанный подграф дерева Кэли $\Im^{k}, k \geqslant 2$, такой, что $|V(K)|=n$. Тогда:

1) $|D(V(K))|=(k-1) n+2$;

2) $|B(V(K))|=k n+2$.

ДокАЗАТЕльство. 1. Воспользуемся методом индукции по $n$. Для $n=1,2$ утверждение тривиально. Предположим, что утверждение верно для $n=m$, т.е. из $|V(K)|=m$ следует, что $|D(V(K))|=(k-1) m+2$. Докажем утверждение для $n=m+1$, т.е. для $\widetilde{K}=K \cup\{x\}$. Так как $\widetilde{K}-$ связанный граф, то $x \in D(V(K))$ и существует единственная точка $y \in S_{1}(x)=\{u \in V: d(x, u)=1\}$ такая, что $y \in V(K)$. Таким образом, $D(V(\widetilde{K}))=(D(V(K)) \backslash\{x\}) \cup\left(S_{1}(x) \backslash\{y\}\right)$. Следовательно,

$$
|D(V(\widetilde{K}))|=|D(V(K))|-1+k=(k-1)(m+1)+2 .
$$

2. Очевидно, что $|B(V(K))|=|V(K)|+|D(V(K))|$. Из этого равенства в силу утверждения 1 леммы мы получим $|B(V(K))|=n+(k-1) n+2=k n+2$.

Лемма 3 [18]. Пусть $G$ - счетный граф с максимальной степенъю $k+1$ (m.е. каждая точка $x \in V(G)$ имеет не более $k+1$ соседей), и пусть $\widetilde{N}_{n, G}(x)$ - число связанных подграфов $G^{\prime} \subset G$ с $x \in V\left(G^{\prime}\right)$ u $\left|E\left(G^{\prime}\right)\right|=n$. Тогда

$$
\widetilde{N}_{n, G}(x) \leqslant(k e)^{n} \text {. }
$$

Для $x \in V$ будем писать $x \in \gamma$, если существует $l \in \gamma$ такое, что $x \in i(l)$ (см. п. 2.1). Обозначим $N_{r}(x)=|\{\gamma: x \in \gamma,|\gamma|=r\}|$.

ЛЕмма 4. Для любого $k \geqslant 2$ имеем

$$
N_{r}(x) \leqslant \alpha^{r}
$$

əде $\alpha=(2 k e)^{4 k+3}$.

ДокАЗАТЕЛЬСтво. Обозначим через $K_{\gamma}$ минимальный связанный подграф дерева $\Im^{k}$, который содержит контур $\gamma=\left\{\gamma_{1}, \ldots, \gamma_{m}\right\}$, где $\gamma_{i}-$ подконтуры, $m \geqslant 1$. 
В силу леммы 2 имеем

$$
\left|B\left(\operatorname{Int} \gamma_{i}\right)\right|=k\left|\operatorname{Int} \gamma_{i}\right|+2=k\left\{\left|D_{\text {int }}\left(\operatorname{Int} \gamma_{i}\right)\right|+\left|\operatorname{Int} \gamma_{i} \backslash D_{\text {int }}\left(\operatorname{Int} \gamma_{i}\right)\right|\right\}+2 .
$$

Используя равенство (см. лемму 2)

$$
\left|\operatorname{Int} \gamma_{i} \backslash D_{\text {int }}\left(\operatorname{Int} \gamma_{i}\right)\right|=\frac{\left|D_{\text {int }}\left(\operatorname{Int} \gamma_{i}\right)\right|-2}{k-1},
$$

получаем

$$
\left|B\left(\operatorname{Int} \gamma_{i}\right)\right|=k \frac{k\left|D_{\text {int }}\left(\operatorname{Int} \gamma_{i}\right)\right|-2}{k-1}+2 \leqslant 2 k\left|D_{\text {int }}\left(\operatorname{Int} \gamma_{i}\right)\right| .
$$

Очевидно, что

$$
\left|D_{\text {int }}\left(\operatorname{Int} \gamma_{i}\right)\right| \leqslant\left|\operatorname{imp} \gamma_{i}\right|=\left|\gamma_{i}\right|
$$

В силу (9) из (8) имеем

$$
\left|B\left(\operatorname{Int} \gamma_{i}\right)\right| \leqslant 2 k\left|\gamma_{i}\right|
$$

Ясно, что

$$
\sum_{i=1}^{m}\left|\gamma_{i}\right| \leqslant|\gamma|+m, \quad\left|B\left(V\left(K_{\gamma}\right)\right)\right| \leqslant \sum_{i=1}^{m}\left|B\left(\operatorname{Int} \gamma_{i}\right)\right|+2 m-2 .
$$

С учетом (9) из (10) получим

$$
B\left(V\left(K_{\gamma}\right)\right)\left|\leqslant 2 k \sum_{i=1}^{m}\right| \gamma_{i}|+2 m-2 \leqslant 2 k| \gamma \mid+2(k+1) m-2 .
$$

Так как $\gamma$ содержит $m$ подконтуров, то $m \leqslant|\gamma|$, следовательно, из (11) имеем

$$
N_{r}(x) \leqslant \sum_{m=1}^{r}\left(\begin{array}{c}
2 k r+2(k+1) m-2 \\
r
\end{array}\right) \tilde{N}_{2 k r+2(k+1) m-2, \Im^{k}}(x) .
$$

В силу леммы 3 и неравенства $\left(\begin{array}{l}n \\ r\end{array}\right) \leqslant 2^{n-1}$ из (12) получим (7).

\section{5. НЕЕДИНСТВЕННОСТЬ МЕРЫ ГИББСА}

Функция $Z_{\Lambda}^{(i)}$ в объеме $\Lambda$ при граничном условии $\omega_{\Lambda}^{(i)}$ определяется как

$$
Z_{\Lambda}^{(i)}=\sum_{\sigma \in \Omega_{\Lambda}} e^{-\beta H\left(\sigma \mid \omega_{\Lambda^{\prime}}^{(i)}\right)}
$$

где $\beta=1 / T$ - обратная температура. Тогда условная мера Гиббса $\mu_{\Lambda}^{(i)}$ в объеме $\Lambda$ при граничном условии $\omega_{\Lambda}^{(i)}$ определяется следующим образом:

$$
\mu_{\Lambda, \beta}^{(i)}(\sigma)=\frac{e^{-\beta H\left(\sigma \mid \omega_{\Lambda^{\prime}}^{(i)}\right)}}{Z_{\Lambda}^{(i)}}, \quad \sigma \in \Omega_{\Lambda}
$$

Следующая лемма дает контурное представление гамильтониана. 
Лемма 5. Гамильтониан $H_{n}\left(\sigma_{n}\right)=H_{V_{n}}\left(\sigma_{n} \mid \omega_{V_{n}^{\prime}}^{(1)} \equiv 1\right)$ (см. (6)) имеет вид

$$
H_{n}\left(\sigma_{n}\right)=\sum_{i=2}^{6}\left(U_{i}-U_{1}\right)\left|\partial_{i}\right|+\left|M_{n}\right| U_{1},
$$

где $\sigma_{n}=\sigma_{V_{n}}^{(1)}, \sigma_{n, b}=\left(\sigma_{n}\right)_{b},\left|\partial_{i}\right|=\sum_{\gamma \in \Gamma}\left|\left\{b \in \operatorname{imp} \gamma: \sigma_{n, b} \in C_{i}\right\}\right|, M_{n}=\{b \in M:$ $\left.b \cap V_{n} \neq \varnothing\right\}$.

ДоказАТЕЛьСТво. Имеем

$$
H_{n}\left(\sigma_{n}\right)=\sum_{b \in M_{n}} U\left(\sigma_{n, b}\right)=\sum_{j=2}^{6} U_{j}\left|\partial_{j}\right|+\left(\left|M_{n}\right|-|\partial|\right) U_{1}
$$

Тогда из равенства $|\partial|=\sum_{j=2}^{6}\left|\partial_{j}\right|$ получим (13).

Лемма 6. Пусть $J \in \tilde{A}_{1}, \gamma-\oint$ фисированный контур и $P(\gamma)=\mu_{n, \beta}^{(1)}\left\{\sigma_{n}: \gamma \in\right.$ $\left.\Gamma\left(\sigma_{n}\right)\right\}$. Тогда

$$
P(\gamma) \leqslant e^{-\beta \lambda_{0}|\gamma|}
$$

где $\lambda_{0}=\min \left\{U \backslash\left\{U_{j}: U_{j}=U^{\min }\right\}\right\}-U^{\min }, U=\left\{U_{1}, \ldots, U_{6}\right\}, U^{\min }=\min \left\{U_{1}, \ldots, U_{6}\right\}$.

ДокАЗАТЕЛЬСтво. Обозначим $\Omega_{\gamma}=\left\{\sigma_{n}: \gamma \subset \Gamma\left(\sigma_{n}\right)\right\}, \Omega_{\gamma}^{0}=\left\{\sigma_{n}: \gamma \cap \Gamma\left(\sigma_{n}\right)=\varnothing\right\}$ и определим отображение $\chi_{\gamma}: \Omega_{\gamma} \rightarrow \Omega_{\gamma}^{0}$ следующим образом:

$$
\chi_{\gamma}\left(\sigma_{n}\right)(x)= \begin{cases}1, & x \in \operatorname{Int} \gamma \\ \sigma_{n}(x), & x \notin \operatorname{Int} \gamma\end{cases}
$$

Для данного $\gamma$ отображение $\chi_{\gamma}$ является взаимно однозначным.

Для $\sigma_{n} \in \Omega_{V_{n}}$ и $j=2, \ldots, 6$ мы имеем $\left|\partial_{j}\left(\sigma_{n}\right)\right|=\left|\partial_{j}\left(\chi_{\gamma}\left(\sigma_{n}\right)\right)\right|+\left|\gamma_{j}\right|$, где $\gamma_{j}=\{b \in$ $\left.\operatorname{imp} \gamma: \sigma_{b} \in C_{j}\right\}$. Следовательно, в силу леммы 5 имеем

$$
\begin{aligned}
P(\gamma) & =\frac{\sum_{\sigma_{n} \in \Omega_{\gamma}} \exp \left\{-\beta \sum_{j=2}^{6}\left(U_{j}-U_{1}\right)\left|\partial_{j}\left(\sigma_{n}\right)\right|\right\}}{\sum_{\tilde{\sigma}_{n}} \exp \left\{-\beta \sum_{j=2}^{6}\left(U_{j}-U_{1}\right)\left|\partial_{j}\left(\tilde{\sigma}_{n}\right)\right|\right\}} \leqslant \\
& \leqslant \frac{\sum_{\sigma_{n} \in \Omega_{\gamma}} \exp \left\{-\beta \sum_{j=2}^{6}\left(U_{j}-U_{1}\right)\left|\partial_{j}\left(\sigma_{n}\right)\right|\right\}}{\sum_{\tilde{\sigma}_{n} \in \Omega_{\gamma}} \exp \left\{-\beta \sum_{j=2}^{6}\left(U_{j}-U_{1}\right)\left|\partial_{j}\left(\chi_{\gamma}\left(\tilde{\sigma}_{n}\right)\right)\right|\right\}} .
\end{aligned}
$$

Так как $J \in \tilde{A}_{1}$, в силу теоремы 2 имеем $G S_{\mathrm{p}}(H)=\left\{\varphi^{(i)}, i=1,2,3\right\}$, следовательно, $U_{j}-U_{1} \geqslant \lambda_{0}>0 \forall j=2,3, \ldots, 6$. Таким образом, из (15) получим (14).

Лемма 7. Для любого $k \geqslant 2$ и для достаточно больиих $\beta$ существуют постоянные $C=C(k, \beta)>0$ такие, что

$$
\mu_{\beta}^{(1)}\left\{\sigma_{\Lambda}:|\gamma|>C \ln |\Lambda| \quad \text { для некоторых } \quad \gamma \subset \Gamma\left(\sigma_{\Lambda}\right)\right\} \rightarrow 0 \quad \text { nри } \quad|\Lambda| \rightarrow \infty .
$$


ДоказАТЕЛЬСтво. Предположим, что выполнено условие $\beta>\lambda_{0}^{-1} \ln \alpha$. Тогда с помощью лемм 4 и 6 получим

$$
\mu_{\beta}^{(1)}\left\{\sigma_{\Lambda}, t \in \gamma,|\gamma|=r\right\} \leqslant N_{r}(t) e^{-\beta r \lambda_{0}} \leqslant \alpha^{r} e^{-\beta r \lambda_{0}},
$$

где $\alpha=(2 k e)^{4 k+3}$,

$$
\begin{aligned}
\mu_{\beta}^{(1)}\left\{\sigma_{\Lambda}, t \in \gamma,|\gamma|>C_{1} \ln |\Lambda|\right\} & \leqslant \sum_{r \geqslant C_{1} \ln |\Lambda|}\left(\alpha e^{-\beta \lambda_{0}}\right)^{r}= \\
& =\frac{\left(\alpha e^{-\beta \lambda_{0}}\right)^{C_{1} \ln |\Lambda|}}{1-\alpha e^{\beta \lambda_{0}}}=\frac{|\Lambda|^{C_{1}\left(\ln \alpha-\beta \lambda_{0}\right)}}{1-\alpha e^{-\beta \lambda_{0}}},
\end{aligned}
$$

где константа $C_{1}$ будет определена позже. Таким образом, мы имеем

$$
\mu_{\beta}^{(1)}\left\{\sigma_{\Lambda},|\gamma|>C_{1} \ln |\Lambda|\right\} \leqslant \frac{|\Lambda|^{C_{1}\left(\ln \alpha-\beta \lambda_{0}\right)+1}}{1-\alpha e^{-\beta \lambda_{0}}} .
$$

Последнее выражение стремится к нулю, если $|\Lambda| \rightarrow \infty$ и $C_{1}>1 /\left(\lambda_{0} \beta-\ln \alpha\right)$.

Лемма 8. Для любого $k \geqslant 2$ имеет место равномерная по $x \in \Lambda$ сходимость:

$$
\mu_{\beta}^{(1)}\{\sigma(\Lambda): \sigma(x)=j\} \rightarrow 0, \quad j \neq 1, \quad \text { npu } \beta \rightarrow \infty .
$$

ДокАЗАТЕЛЬСтво. Если $\sigma(x)=j$, то $x$ - точка внутри некоторого контура, мы запишем это в виде $x \in \operatorname{Int}_{j} \gamma$. Предположим $t \in \gamma$ и $x \in \operatorname{Int}_{j} \gamma$, тогда $|\gamma| \geqslant|t|+2$. Следовательно,

$$
\begin{gathered}
\mu_{\beta}^{(1)}\left\{\sigma_{\Lambda}: x \in \operatorname{Int}_{j} \gamma, t \in \gamma,|\gamma|<C_{1} \ln |\Lambda|\right\} \leqslant \frac{\left(\alpha e^{-\beta \lambda_{0}}\right)^{|t|+2}}{1-\alpha e^{-\beta \lambda_{0}}}, \\
\mu_{\beta}^{(1)}\{\sigma(x)=j\} \leqslant \mu_{\beta}^{(1)}\left\{\sigma_{\Lambda}: x \in \operatorname{Int}_{j} \gamma, \gamma \subset \Gamma\left(\sigma_{\Lambda}\right)\right\} \leqslant \sum_{|t|=1}^{C_{1} \ln |\Lambda|} \frac{\left(\alpha e^{-\beta \lambda_{0}}\right)^{|t|+2}}{1-\alpha e^{-\beta \lambda_{0}}}+ \\
\quad+\mu_{\beta}^{(1)}\left\{\sigma_{\Lambda}: \exists \quad \text { контур } \gamma \subset \Gamma\left(\sigma_{\Lambda}\right) \quad \text { такой, что }|\gamma| \geqslant C_{1} \ln |\Lambda|\right\} \leqslant \\
\leqslant \frac{\left(\alpha e^{-\beta \lambda_{0}}\right)^{3}}{\left(1-\alpha e^{-\beta \lambda_{0}}\right)^{2}}+\frac{|\Lambda|^{C_{1}\left(\ln \alpha-\beta \lambda_{0}\right)+1}}{1-\alpha e^{-\beta \lambda_{0}}} .
\end{gathered}
$$

При $|\Lambda| \rightarrow \infty$ и $\beta \rightarrow \infty$ мы получим $\mu_{\beta}^{(1)}\{\sigma(x)=j\} \rightarrow 0, j \neq 1$.

Теорема 4. Для всех достаточно больших $\beta$ существуют по крайней мере три меры Гиббса для модели Поттса при $q=3$ с конкурирующими взаимодействиями на дереве Кэли порлдка $k \geqslant 2$.

ДокаЗАтЕЛЬСтво. Заметим, что аналог леммы 8 справедлив при $i=2,3$, т.е. для $\mu_{\beta}^{(i)}$. Следовательно, с помощью леммы 8 мы имеем

$$
\lim _{\beta \rightarrow \infty} \mu_{\beta}^{(i)}\{\sigma(x)=j\}= \begin{cases}0, & i \neq j, \\ 1, & i=j,\end{cases}
$$

откуда для любых $i, j \in\{1,2,3\}, i \neq j$, и достаточно больших $\beta$ получаем

$$
\mu_{\beta}^{(i)}\{\sigma(x)=i\} \neq \mu_{\beta}^{(j)}\{\sigma(x)=i\} .
$$

Теорема доказана. 
Благодарности. У. А. Розиков благодарит NATO Reintegration Grant (FEL.RIG. 980771) и Institute des Hautes Etudes Scientiques (Бюр-сюр-Ивет, Франция) за финансовую поддержку пребывания в IHES в октябре-декабре 2006 г., а также International Center for Theoretical Physics (Триест, Италия) за финансовую поддержку пребывания в ICTP в мае-августе 2006 г. Авторы выражают глубокую признательность профессорам М. Кассандро, М. Концевичу, Ю. Сухову и Ф. Мухамедову за полезную дискуссию.

\section{Список литературы}

[1] U. A. Rozikov, Lett. Math. Phys., 71 (2005), 27-38.

[2] U. A. Rozikov, J. Stat. Phys., 122 (2006), 217-235.

[3] R. Fernández, Contour ensembles and the description of Gibbsian probability distributions at low temperature, LMRS/persopage/Fernandez/cont.ps.gz.

[4] R. A. Minlos, Introduction to Mathematical Statistical Physics, Univ. Lecture Ser., 19, AMS, Providence, RI, 2000.

[5] С. А. Пирогов, Я. Г. Синай, ТМФ, 25:3 (1975), 358-369; 26:1 (1976), 61-76.

[6] Я. Г. Синай, Теория фазовых переходов. Строгие результаты, Наука, М., 1980.

[7] M. Zahradnik, Commun. Math. Phys., 93 (1984), 559-581.

[8] M. Zahradnik, Rend. Mat. Appl. (7), 18 (1998), 411-486.

[9] R. Peierls, Proc. Cambridge Philos. Soc., 32 (1936), 477-481.

[10] П. М. Блехер, Н. Н. Ганиходжаев, Теория вероят. и ее примен., 35:2 (1990), 220-230.

[11] F. M. Mukhamedov, U. A. Rozikov, J. Stat. Phys., 114 (2004), 825-848.

[12] У. А. Розиков, ТМФ, 112:1 (1997), 170-175.

[13] U. A. Rozikov, Yu. M. Suhov, Queueing Syst., 46 (2004), 197-212.

[14] Р. Бэкстер, Точно решаемые модели в статистической физике, Мир, М., 1985.

[15] W. Holsztynski, J. Slawny, Commun. Math. Phys., 61 (1978), 177-190.

[16] И. А. Кашапов, ТМФ, 33:1 (1977), 110-118.

[17] Н. Н. Ганиходжаев, У. А. Розиков, ТМФ, 111:1 (1997), 109-117.

[18] C. Borgs, Statistical physics expansion methods in combinatorics and computer science, http://research.microsoft. com/ borgs/CBMS.pdf.

Поступила в редакцию 11.12.2006

4 Теоретическая и математическая физика, т. 153, № 1, 2007 г. 